\title{
On Growth of Meromorphic Solutions for Linear Difference Equations
}

\author{
Zong-Xuan Chen ${ }^{1}$ and Kwang Ho Shon ${ }^{2}$ \\ ${ }^{1}$ School of Mathematical Sciences, South China Normal University, Guangzhou 510631, China \\ ${ }^{2}$ Department of Mathematics, College of Natural Sciences, Pusan National University, Pusan 609-735, Republic of Korea
}

Correspondence should be addressed to Kwang Ho Shon; khshon@pusan.ac.kr

Received 9 June 2013; Accepted 19 August 2013

Academic Editor: Norio Yoshida

Copyright (c) 2013 Z.-X. Chen and K. H. Shon. This is an open access article distributed under the Creative Commons Attribution License, which permits unrestricted use, distribution, and reproduction in any medium, provided the original work is properly cited.

We mainly study growth of linear difference equations $P_{n}(z) f(z+n)+\cdots+P_{1}(z) f(z+1)+P_{0}(z) f(z)=0$ and $P_{n}(z) f(z+n)+$ $\cdots+P_{1}(z) f(z+1)+P_{0}(z) f(z)=F(z)$, where $F(z), P_{0}(z), \ldots, P_{n}(z)$ are polynomials such that $F(z) P_{0}(z) P_{n}(z) \not \equiv 0$ and give the most weak condition to guarantee that orders of all transcendental meromorphic solutions of the above equations are greater than or equal to 1.

\section{Introduction and Results}

Consider growth of meromorphic solutions of the following linear difference equations:

$$
P_{n}(z) f(z+n)+\cdots+P_{1}(z) f(z+1)+P_{0}(z) f(z)=0,
$$

$P_{n}(z) f(z+n)+\cdots+P_{1}(z) f(z+1)+P_{0}(z) f(z)=F(z)$,

where $F(z), P_{0}(z), \ldots, P_{n}(z)$ are polynomials such that $F(z)$ $P_{0}(z) P_{n}(z) \not \equiv 0$

Recently, several papers (including [1-8]) have been published regarding growth of the solutions of (1) and (2). We recall the following results. Ishizaki and Yanagihara proved the following theorem.

Theorem A (see [5]). Let $f(z)$ be a transcendental entire solution of

$$
Q_{n}(z) \Delta^{n} f(z)+\cdots+Q_{1}(z) \Delta f(z)+Q_{0}(z) f(z)=0,
$$

where $Q_{n}, \ldots, Q_{0}$ are polynomials, $\Delta f(z)=f(z+1)-f(z)$, $\Delta^{n} f(z)=\Delta\left(\Delta^{n-1} f(z)\right)$, and of order $\chi<1 / 2$. Then one has

$$
\log M(r, f)=\operatorname{Lr} r^{\chi}(1+o(1)),
$$

where a rational number $\chi$ is a slope of the Newton polygon for (3) and $L>0$ is a constant. In particular, one has $\chi>0$.

Remark 1. In [5], Ishizaki and Yanagihara give an example. The difference equation

$$
\begin{aligned}
& \left(6 z^{2}+19 z+15\right) \Delta^{3} f(z)+(z+3) \Delta^{2} f(z) \\
& -\Delta f(z)-f(z)=0,
\end{aligned}
$$

that is,

$$
\begin{aligned}
& \left(6 z^{2}+19 z+15\right) f(z+3)-\left(18 z^{2}+56 z+42\right) f(z+2) \\
& \quad+\left(18 z^{2}+55 z+38\right) f(z+1)-\left(6 z^{2}+18 z+12\right) f(z) \\
& =0
\end{aligned}
$$

admits an entire solution of order $1 / 3$.

In [5], Ishizaki and Yanagihara do not give a concrete solution of order $1 / 3$. In fact, we assert that (5) has no entire solution of order $1 / 3$. Contrary to the assertion, we assume that $f(z)$ is an entire solution of order $1 / 3$ of $(5)^{\prime}$. Set 
$g(z)=f(z)-1$. Then $g(z)$ is an entire function of order $1 / 3$. Substituting $f(z)=g(z)+1$ in to $(5)^{\prime}$, we obtain

$$
\begin{aligned}
\left(6 z^{2}+\right. & 19 z+15) g(z+3)-\left(18 z^{2}+56 z+42\right) g(z+2) \\
& +\left(18 z^{2}+55 z+38\right) g(z+1) \\
& -\left(6 z^{2}+18 z+12\right) g(z)=1 .
\end{aligned}
$$

Using the same method as in the proof of Case 1, in proof of Theorem 4, we obtain the order of $g$ which is greater than or equal to 1 . It is a contradiction.

Thus, we determine that whether (1) ((2) or (3)) has a transcendental meromorphic solution of order $<1$, it becomes a significant problem for mathematicians.

Chiang and Feng proved the following theorem.

Theorem B (see [3]). Let $P_{0}(z), \ldots, P_{n}(z)$ be polynomials such that there exists an integer $l, 0 \leq l \leq n$ so that

$$
\operatorname{deg}\left(P_{l}\right)>\max _{0 \leq j \leq n, j \neq l}\left\{\operatorname{deg}\left(P_{j}\right)\right\}
$$

holds. Suppose that $f(z)$ is a meromorphic solution of (1). Then, one has $\sigma(f) \geq 1$.

In this paper, we use the basic notions of Nevanlinna's theory (see $[9,10])$. In addition, we use the notations $\sigma(f)$ to denote the order of growth of a meromorphic function $f(z)$ and $\lambda(f)$ to denote the exponent of convergence of zeros of $f(z)$.

Remark 2. Comparing Theorem A with Theorem B, we see that since (3) can be rewritten as (1), Theorem A shows that, under general case, (1) may have transcendental meromorphic solution $f(z)$ with $\sigma(f)<1$. In Theorem B, the condition (7) guarantees that all meromorphic solutions of (1) satisfy $\sigma(f) \geq 1$.

The author who weakened the condition (7) of Theorem $\mathrm{B}$ proved the following results.

Theorem C (see [2]). Let $P_{n}(z), \ldots, P_{0}(z)$ be polynomials such that $P_{n} P_{0} \neq \equiv 0$ and

$$
\operatorname{deg}\left(P_{n}+\cdots+P_{0}\right)=\max \left\{\operatorname{deg} P_{j}: j=0, \ldots, n\right\} \geq 1 .
$$

Then every finite-order meromorphic solution $f(z)(\not \equiv 0)$ of $(1)$ satisfies $\sigma(f) \geq 1, f(z)$ assumes every nonzero value $a \in \mathbb{C}$ infinitely often, and $\lambda(f-a)=\sigma(f)$.

Theorem D (see [2]). Let $F(z), P_{n}(z), \ldots, P_{0}(z)$ be polynomials such that $F P_{n} P_{0} \quad \neq \quad 0$ and (8). Then every finiteorder transcendental meromorphic solution $f(z)$ of (2) satisfies $\sigma(f) \geq 1$ and $\lambda(f)=\sigma(f)$.

Theorem E (see [2]). Let $F(z), P_{n}(z), \ldots, P_{0}(z)$ be polynomials such that $F P_{n} P_{0} \neq \equiv$. Suppose that $f(z)$ is a meromorphic solution with infinitely many poles of (1) (or (2)). Then $\sigma(f) \geq$ 1 .
From $(5)^{\prime}$ we see that the sum of coefficients of $(5)^{\prime}$, which is equal to -1 , does not satisfy the condition (8), but all transcendental entire solutions of $(5)^{\prime}$ have order $\sigma(f) \geq 1$.

Thus, a natural question to ask is whether the condition (8) can be weakened.

In this note, we consider this question, again weaken the condition (8) and prove the following results.

Theorem 3. Let $P_{n}(z), \ldots, P_{0}(z)$ be polynomials such that $P_{n} P_{0} \not \equiv 0$ and satisfy

$$
P_{n}(z)+\cdots+P_{0}(z) \not \equiv 0 .
$$

Then every-finite order transcendental meromorphic solution $f(z)(\equiv \quad 0)$ of $(1)$ satisfies $\sigma(f) \geq 1, f(z)$ assumes every nonzero value $a \in \mathbb{C}$ infinitely often, and $\lambda(f-a)=\sigma(f)$.

Theorem 4. Let $F(z), P_{n}(z), \ldots, P_{0}(z)$ be polynomials such that $F P_{n} P_{0} \quad \neq \quad 0$. Then every finite-order transcendental meromorphic solution $f(z)$ of $(2)$ satisfies $\lambda(f)=\sigma(f) \geq 1$.

Remark 5. For the homogeneous equation (1) by Theorems B, $\mathrm{C}$ and 3, we see that the condition (9) is weaker than (7) and (8). For the nonhomogeneous equation (2) by Theorem 4, we see that the condition (9) is omitted. But, under the condition (8), (1) has no nonzero rational solution, and under the condition (9), (1) may have nonzero rational solution. For example,

$$
(z+1) f(z+1)-z f(z)=0,
$$

has a rational solution $f(z)=1 / z$. This shows that Theorem $\mathrm{C}$ can not be replaced by Theorem 3 completely.

Example 6. The equation

$$
\left(\frac{1}{2} z-1\right) f(z+2)-2(z-2) f(z)=0
$$

has a solution $f(z)=2^{z}$; here $f(z)$ satisfies $\lambda(f-a)=$ $\sigma(f)=1$ for any nonzero finite value $a$, and $f(z)$ has no zero. This shows that in Theorem 3 , the condition $a \neq 0$ cannot be omitted.

Example 7. The equation

$$
f(z+2)+f(z+1)-\left(z^{2}+2 z\right) f(z)=-z^{2}-2 z+2,
$$

has a solution $f(z)=\Gamma(z)+1$ which satisfies $\lambda(f-1)=0$. This shows that in Theorem 4 a solution of (2) does not satisfy $\lambda(f-a)=\sigma(f)$ for a nonzero constant $a$.

By Theorem 3, we can obtain the following corollary.

Corollary 8. Let $P_{n}(z), \ldots, P_{0}(z)$ be polynomials such that $P_{n} P_{0} \not \equiv 0$. If (1) has a transcendental meromorphic solution $f$ with $\sigma(f)<1$, then

$$
P_{n}(z)+\cdots+P_{0}(z) \equiv 0 .
$$

Consider the growth of the second order linear difference equation

$$
\Delta^{2} y(z)+A(z) y(z)=0
$$


with $A(z)$ is a meromorphic function. Since (14) is closely related with the difference Riccati equation

$$
\Delta f(z)+\frac{f(z)^{2}+A(z)}{f(z)-1}=0,
$$

we see that (14) is an important linear difference equation (see [4]).

Ishizaki [4] proved the following result.

Theorem F (see [4]). Suppose that $A(z)$ is a rational function in (14) and has no transcendental meromorphic solutions of order less than 1/2. Further, one assumes that (14) possesses a rational solution. Then, every transcendental meromorphic solution of (14) has order of at least 1.

In this note, we improve this result, omit the condition of Theorem F "(14) possesses a rational solution", and prove the same result.

Theorem 9. Let $A(z)$ be a rational function. Then every transcendental meromorphic solution of (14) has order of at least 1 .

Further, If $A(z)=P(z) / Q(z)$, where $P$ and $Q$ are nonconstant polynomials such that $\operatorname{deg} P \geq \operatorname{deg} Q$, then (14) has no nonzero rational solution.

For the linear difference equation with transcendental coefficients, one has

$$
A_{n}(z) f(z+n)+\cdots+A_{1}(z) f(z+1)+A_{0}(z) f(z)=0 .
$$

Chiang and Feng proved the following result.

Theorem G (see [3]). Let $A_{0}(z), \ldots, A_{n}(z)$ be entire functions such that there exists an integer $l, 0 \leq l \leq n$, such that

$$
\sigma\left(A_{l}\right)>\max \left\{\sigma\left(A_{j}\right): 0 \leq j \leq n, j \neq l\right\} .
$$

If $f(z)(\equiv 0)$ is a meromorphic solution of (16), then one has $\sigma(f) \geq \sigma\left(A_{l}\right)+1$.

Laine and Yang [6] prove that if $A_{0}(z), \ldots, A_{n}(z)$ are entire functions of finite order so that among those having the maximal order $\sigma:=\max \left\{\sigma\left(A_{k}\right): 0 \leq k \leq n\right\}$, exactly one has its type strictly greater than the others, then every meromorphic solution of (16) satisfies $\sigma(f) \geq \sigma+1$.

Remark 10. If $A_{j}(j=0, \ldots, n)$ are meromorphic functions satisfying (17), then Theorem $\mathrm{G}$ does not hold. For example,

$$
y(z+1)-\left(e^{i}+\frac{e^{i}-1}{e^{i z}-1}\right) y(z)=0,
$$

has a solution $y(z)=e^{i z}-1$, in which $\sigma(y)=1<\sigma\left(A_{0}\right)+1$.

This example shows that for the linear difference equation with meromorphic coefficients, the condition (17) can not guarantee that every transcendental meromorphic solution $f(z)$ of (16) satisfies $\sigma(f) \geq \sigma\left(A_{l}\right)+1$.
Thus, a natural question to ask is what conditions will guarantee that every transcendental meromorphic solution $f(z)$ of (16) satisfies $\sigma(f) \geq \sigma\left(A_{l}\right)+1$.

We answer this question and prove the following result.

Theorem 11. Let $A_{0}(z), \ldots, A_{n}(z)$ be meromorphic functions such that there exists an integer $l, 0 \leq l \leq n$, such that

$$
\sigma\left(A_{l}\right)>\max \left\{\sigma\left(A_{j}\right): 0 \leq j \leq n, j \neq l\right\}, \quad \delta\left(\infty, A_{l}\right)>0 .
$$

If $f(z)(\equiv \equiv 0)$ is a meromorphic solution of (16), then one has $\sigma(f) \geq \sigma\left(A_{l}\right)+1$.

\section{Proofs of Theorems}

We need following lemmas and remark to prove Theorems 3, 4,9 , and 11 .

Remark 12. Following Hayman [11, p. 75-76], we define an $\varepsilon$ set to be a countable union of open discs not containing the origin and subtending angles at the origin whose sum is finite. If $E$ is an $\varepsilon$-set, then the set of $r \geq 1$ for which the circle $S(0, r)$ meets $E$ has finite logarithmic measure, and for almost all real $\theta$ the intersection of $E$ with the ray $\arg z=\theta$ is bounded.

Lemma 13 (see [12]). Let $g$ be a function transcendental and meromorphic in the plane of order less than 1 . Let $h>0$. Then there exists an $\varepsilon$-set $E$ such that

$$
\frac{g^{\prime}(z+c)}{g(z+c)} \longrightarrow 0, \quad \frac{g(z+c)}{g(z)} \longrightarrow 1 \text { as } z \longrightarrow \infty \text { in } \mathbb{C} \backslash E,
$$

uniformly in $c$ for $|c| \leq h$. Further, E may be chosen so that for large $z$ not in $E$ the function $g$ has no zeros or poles in $|\zeta-z| \leq h$.

Lemma 14 (see $[6,13])$. Let $w(z)$ be a nonconstant finite-order meromorphic solution of

$$
P(z, w)=0
$$

where $P(z, w)$ is a difference polynomial in $w(z)$. If $P(z, a) \not \equiv 0$ for a meromorphic function $a(z)$ satisfying $T(r, a)=S(r, w)$, then

$$
m\left(r, \frac{1}{w-a}\right)=S(r, w)
$$

Lemma 15 (see $[3,13])$. Given two distinct complex constants $\eta_{1}, \eta_{2}$, let $f$ be a meromorphic function of finite order $\sigma$. Then, for each $\varepsilon>0$, one has

$$
m\left(r, \frac{f\left(z+\eta_{1}\right)}{f\left(z+\eta_{2}\right)}\right)=O\left(r^{\sigma-1+\varepsilon}\right)
$$


Proof of Theorem 4. Suppose that $f(z)$ is a transcendental meromorphic solution of $(2)$ with $\sigma(f)<\infty$. We divide this proof into the following two cases.

Case 1. Suppose that $f(z)$ has only finitely many poles. Now we suppose that $\sigma(f)<1$. By Lemma 13, there exists an $\varepsilon$-set $E$ such that

$$
\begin{gathered}
f(z+j)=f(z)\left(1+o_{j}(1)\right) \\
j=1, \ldots, n \text { as } z \longrightarrow \infty \text { in } \mathbb{C} \backslash E,
\end{gathered}
$$

where $o_{j}(1)(j=1, \ldots, n)$ satisfy

$$
o_{j}(1) \longrightarrow 0 \quad \text { as } z \longrightarrow \infty \text { in } \mathbb{C} \backslash E .
$$

Set $H=\{|z|=r: z \in E,|z|>1\}$. By Remark $12, H$ is of finite logarithmic measure. Substituting (24) into (2), we obtain, as $z \rightarrow \infty$ in $\mathbb{C} \backslash E$,

$$
\begin{aligned}
P_{n}(z) & f(z)\left(1+o_{n}(1)\right)+\cdots+P_{1}(z) f(z)\left(1+o_{1}(1)\right) \\
+ & P_{0}(z) f(z)=F(z),
\end{aligned}
$$

that is,

$$
f(z)=\frac{F(z)}{P_{n}(z)\left(1+o_{n}(1)\right)+\cdots+P_{1}(z)\left(1+o_{1}(1)\right)+P_{0}(z)} .
$$

Thus, since $f(z)$ has only finitely many poles, we deduce that when $|z|=r \notin H$,

$$
\begin{aligned}
& T(r, f) \\
&=m(r, f)+N(r, f)=m(r, f)+O(\log r) \\
&=m\left(r, \frac{F(z)}{P_{n}(z)\left(1+o_{n}(1)\right)+\cdots+P_{0}(z)}\right) \\
& \quad+O(\log r) \\
& \leq T\left(r, \frac{F(z)}{P_{n}(z)\left(1+o_{n}(1)\right)+\cdots+P_{0}(z)}\right) \\
& \quad+O(\log r) \\
& \leq T(r, F)+\sum_{j=0}^{n} T\left(r, P_{j}\right)+\sum_{j=1}^{n} T\left(r, 1+o_{j}(1)\right)+O(\log r) \\
&= O(\log r) .
\end{aligned}
$$

This contradicts with the fact that $f$ is transcendental. Hence $\sigma(f) \geq 1$.

Case 2. Suppose that $f(z)$ has infinitely many poles. Thus, by Theorem $\mathrm{D}$, we see that $\sigma(f) \geq 1$.

Finally, we prove that $\lambda(f)=\sigma(f)$. By (2), and we set

$$
E(z, f):=P_{n}(z) f(z+n)+\cdots+P_{0}(z) f(z)-F(z) .
$$

Thus,

$$
E(z, 0)=F(z) \not \equiv 0 .
$$

By Lemma 14, we have

$$
m\left(r, \frac{1}{f}\right)=S(r, f),
$$

so that

$$
N\left(r, \frac{1}{f}\right)=T(r, f)+S(r, f) .
$$

Hence $\lambda(f)=\sigma(f)$.

Thus, Theorem 4 is proved.

Proof of Theorem 3. Suppose that $f$ is a transcendental meromorphic solution of (1) with $\sigma(f)<\infty$ and that $d \neq 0$ is a constant. Set $g(z)=f(z)-d$. Then, $\sigma(g)=\sigma(f)$.

Substituting $f(z)=g(z)+d$ into (1), we obtain

$$
\begin{aligned}
P_{n}(z) & g(z+n)+\cdots+P_{1}(z) g(z+1)+P_{0}(z) g(z) \\
= & -d\left(P_{n}(z)+\cdots+P_{1}(z)+P_{0}(z)\right) .
\end{aligned}
$$

Since $P_{n}(z)+\cdots+P_{1}(z)+P_{0}(z) \not \equiv 0$, we see that (33) satisfies the conditions of Theorem 4 . Thus, we deduce that $\sigma(g)=$ $\sigma(f) \geq 1$

Finally, we prove that $f(z)$ assumes every nonzero value $a \in \mathbb{C}$ infinitely often and that $\lambda(f-a)=\sigma(f)$. Set

$$
E(z, f):=P_{n}(z) f(z+n)+\cdots+P_{0}(z) f(z) .
$$

Thus, since $a \neq 0$ and (9), we have

$$
E(z, a)=a\left(P_{n}(z)+\cdots+P_{0}(z)\right) \not \equiv 0 .
$$

By Lemma 14 and (35), we have

$$
m\left(r, \frac{1}{f-a}\right)=S(r, f)
$$

so that

$$
N\left(r, \frac{1}{f-a}\right)=T(r, f)+S(r, f) .
$$

Hence $\lambda(f-a)=\sigma(f)$. Theorem 3 is thus proved.

Proof of Theorem 9. Suppose that $y(z)$ is a transcendental meromorphic solution of (14). We rewrite (14) as

$$
y(z+2)-2 y(z+1)+(A+1) y(z)=0
$$

If $A(z)=-1$, then by (38), we obtain

$$
y(z+2)-2 y(z+1)=0 .
$$

We affirm that $\sigma(y) \geq 1$. In fact, if $\sigma(y)<1$, then $y(z)$ has infinitely many zeros, or infinitely many poles. If $y(z)$ has infinitely many zeros, then by (39), we see that if $z_{0}$ is a zero of $y(z)$, then $z_{0}+n, n=1, \ldots$, are also zeros of $y(z)$. Thus, 
$\sigma(y) \geq 1$. If $y(z)$ has infinitely many poles, then by using the same method, we can obtain $\sigma(y) \geq 1$.

Now we suppose that $A(z) \neq-1$. Set $A(z)=P(z) / Q(z)$, where $P(z)$ and $Q(z)$ are nonzero polynomials. By (38), we have

$$
Q(z) y(z+2)-2 Q(z) y(z+1)+(P(z)+Q(z)) y(z)=0 \text {. }
$$

Since

$$
Q(z)+(-2 Q(z))+(P(z)+Q(z))=P(z) \not \equiv 0,
$$

by Theorem 3 , we see that $\sigma(y) \geq 1$.

Further, if $P$ and $Q$ are nonconstant polynomials such that $\operatorname{deg} P \geq \operatorname{deg} Q$, then (41) satisfies the condition of Theorem C. Thus, we see that (14) has no rational solution. Thus, Theorem 9 is proved.

Proof of Theorem 11. Clearly, (16) has no nonzero rational solution.

Now suppose that $f(z)$ is a transcendental meromorphic solution of (16) with $\sigma(f)<\infty$. By (16), we obtain

$$
\begin{aligned}
-A_{l}= & A_{n} \frac{f(z+n)}{f(z+l)}+\cdots+A_{l+1} \frac{f(z+l+1)}{f(z+l)} \\
& +A_{l-1} \frac{f(z+l-1)}{f(z+l)}+\cdots+A_{0} \frac{f(z)}{f(z+l)} .
\end{aligned}
$$

Set

$$
\begin{gathered}
\max \left\{\sigma\left(A_{j}\right): 0 \leq j \leq n, j \neq l\right\}=s<\sigma\left(A_{l}\right)=\sigma \\
\delta\left(\infty, A_{l}\right)=\delta>0 .
\end{gathered}
$$

Thus, we have

$$
m\left(r, A_{l}\right)>\frac{1}{2} \delta T\left(r, A_{l}\right)
$$

By Lemma 15, we see that for given $\varepsilon(0<3 \varepsilon<\sigma-s)$,

$$
m\left(r, \frac{f(z+j)}{f(z+l)}\right)=O\left(r^{\sigma(f)-1+\varepsilon}\right), \quad(0 \leq j, l \leq n, j \neq l) .
$$

Thus, by (42), (45), and (46), we have

$$
\begin{aligned}
\frac{1}{2} \delta T & \left(r, A_{l}\right) \\
& \leq m\left(r, A_{l}\right) \\
& \leq \sum_{0 \leq j \leq n, j \neq l} m\left(r, A_{j}\right)+\sum_{0 \leq j \leq n, j \neq l} m\left(r, \frac{f(z+j)}{f(z+l)}\right) \\
& \leq \sum_{0 \leq j \leq n, j \neq l} T\left(r, A_{j}\right)+\sum_{0 \leq j \leq n, j \neq l} O\left(r^{\sigma(f)-1+\varepsilon}\right) \\
& \leq \sum_{0 \leq j \leq n, j \neq l} T\left(r, A_{j}\right)+O\left(r^{\sigma(f)-1+\varepsilon}\right) .
\end{aligned}
$$

By (43), we see that for given $\varepsilon$ above,

$$
T\left(r, A_{j}\right)<r^{s+\varepsilon}, \quad(0 \leq j \leq n, j \neq l) .
$$

Since $\sigma\left(A_{l}\right)=\sigma$, we see that there is a sequence $r_{j}\left(1<r_{1}<\right.$ $\left.r_{2}<\cdots, r_{j} \rightarrow \infty\right)$ satisfying

$$
T\left(r_{j}, A_{l}\right)>r_{j}^{\sigma-\varepsilon} \text {. }
$$

Thus, by (47)-(49), we obtain

$$
\frac{1}{2} \delta r_{j}^{\sigma-\varepsilon} \leq(n-1) r_{j}^{s+\varepsilon}+M r_{j}^{\sigma(f)-1+\varepsilon}
$$

If we combine this with $3 \varepsilon<\sigma-s$, it follows that

$$
\frac{1}{2} \delta r_{j}^{\sigma-\varepsilon}(1+o(1)) \leq M r_{j}^{\sigma(f)-1+\varepsilon}
$$

So that, it follows that $\sigma(f) \geq \sigma+1$. Thus, Theorem 11 is proved.

\section{Acknowledgments}

Zong-Xuan Chen was supported by the National Natural Science Foundation of China (no. 11171119). Kwang Ho Shon was supported by Basic Science Research Program through the National Research Foundation of Korea (NRF) funded by the Ministry of Science, ICT and Future Planning (2013R1A1A2008978).

\section{References}

[1] Z. Chen, "On growth, zeros and poles of meromorphic solutions of linear and nonlinear difference equations," Science China, vol. 54, no. 10, pp. 2123-2133, 2011.

[2] Z.-X. Chen, "Growth and zeros of meromorphic solution of some linear difference equations," Journal of Mathematical Analysis and Applications, vol. 373, no. 1, pp. 235-241, 2011.

[3] Y.-M. Chiang and S.-J. Feng, "On the Nevanlinna characteristic of $f(z+\eta)$ and difference equations in the complex plane," Ramanujan Journal, vol. 16, no. 1, pp. 105-129, 2008.

[4] K. Ishizaki, "On difference Riccati equations and second order linear difference equations," Aequationes Mathematicae, vol. 81, no. 1-2, pp. 185-198, 2011.

[5] K. Ishizaki and N. Yanagihara, "Wiman-Valiron method for difference equations," Nagoya Mathematical Journal, vol. 175, pp. 75-102, 2004.

[6] I. Laine and C.-C. Yang, "Clunie theorems for difference and q-difference polynomials," Journal of the London Mathematical Society. Second Series, vol. 76, no. 3, pp. 556-566, 2007.

[7] S. Li and Z.-S. Gao, "Finite order meromorphic solutions of linear difference equations," Proceedings of the Japan Academy Series A, vol. 87, no. 5, pp. 73-76, 2011.

[8] X.-M. Zheng and J. Tu, "Growth of meromorphic solutions of linear difference equations," Journal of Mathematical Analysis and Applications, vol. 384, no. 2, pp. 349-356, 2011.

[9] W. K. Hayman, Meromorphic Functions, Oxford Mathematical Monographs, Clarendon Press, Oxford, UK, 1964.

[10] I. Laine, Nevanlinna Theory and Complex Differential Equations, vol. 15 of de Gruyter Studies in Mathematics, Walter de Gruyter \& Co., Berlin, Germany, 1993. 
[11] W. K. Hayman, "Slowly growing integral and subharmonic functions," Commentarii Mathematici Helvetici, vol. 34, pp. 7584, 1960.

[12] W. Bergweiler and J. K. Langley, "Zeros of differences of meromorphic functions," Mathematical Proceedings of the Cambridge Philosophical Society, vol. 142, no. 1, pp. 133-147, 2007.

[13] R. G. Halburd and R. J. Korhonen, "Difference analogue of the lemma on the logarithmic derivative with applications to difference equations," Journal of Mathematical Analysis and Applications, vol. 314, no. 2, pp. 477-487, 2006. 


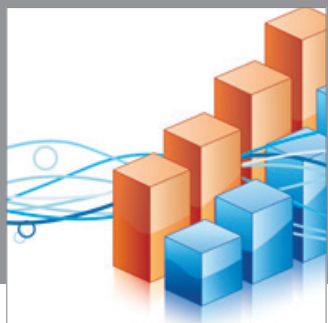

Advances in

Operations Research

mansans

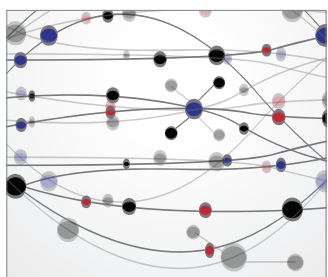

The Scientific World Journal
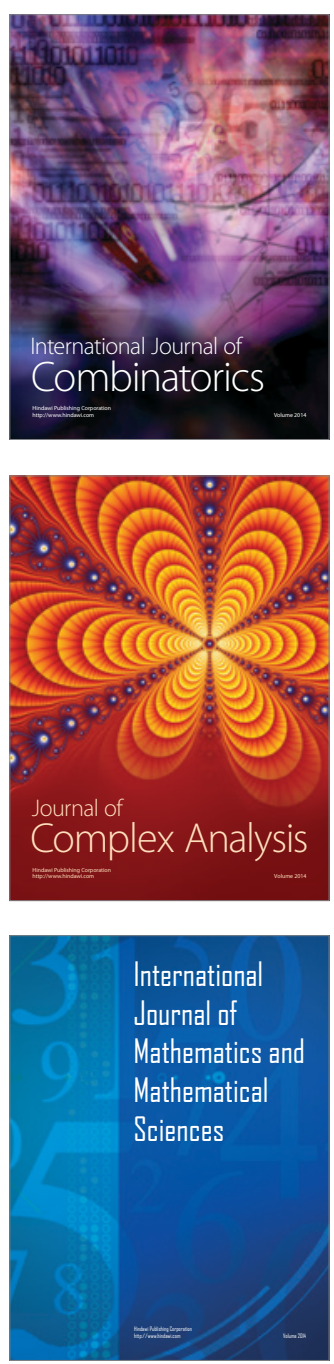
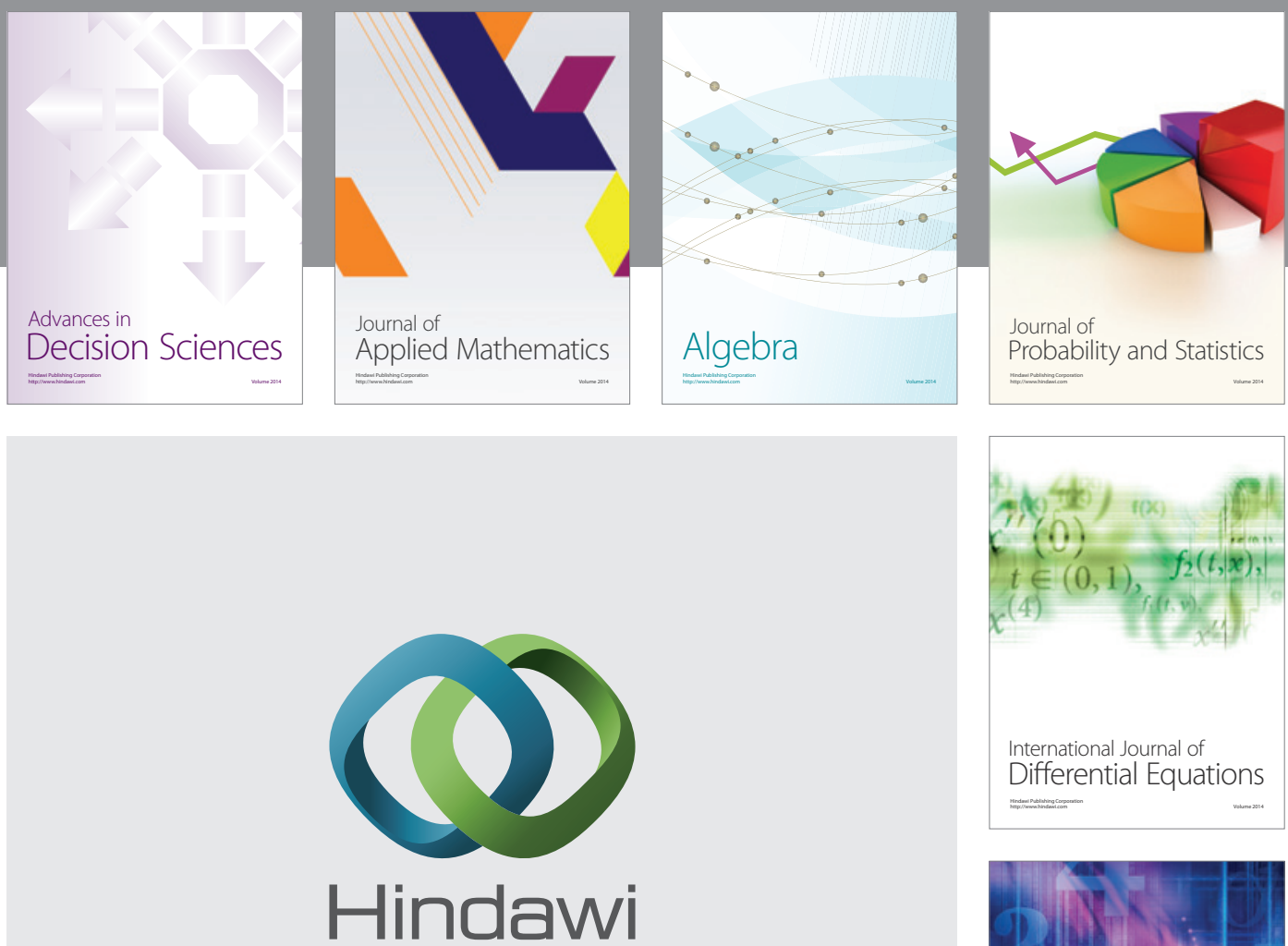

Submit your manuscripts at http://www.hindawi.com
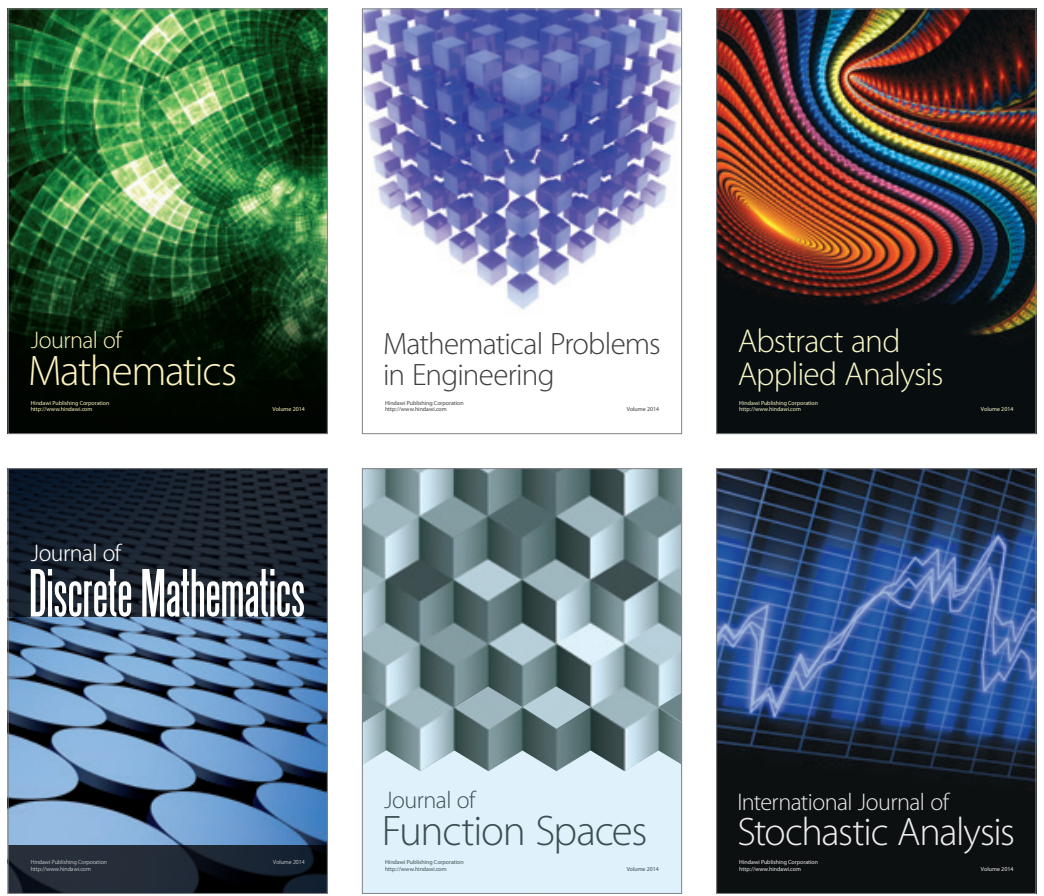

Journal of

Function Spaces

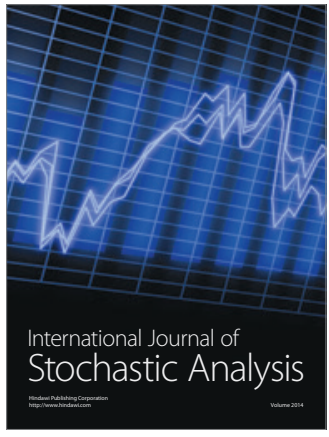

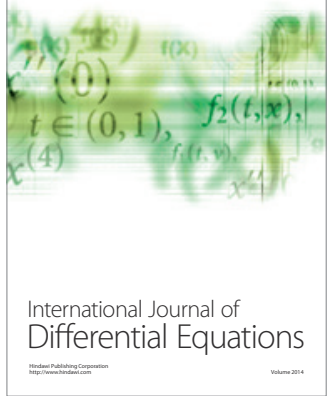
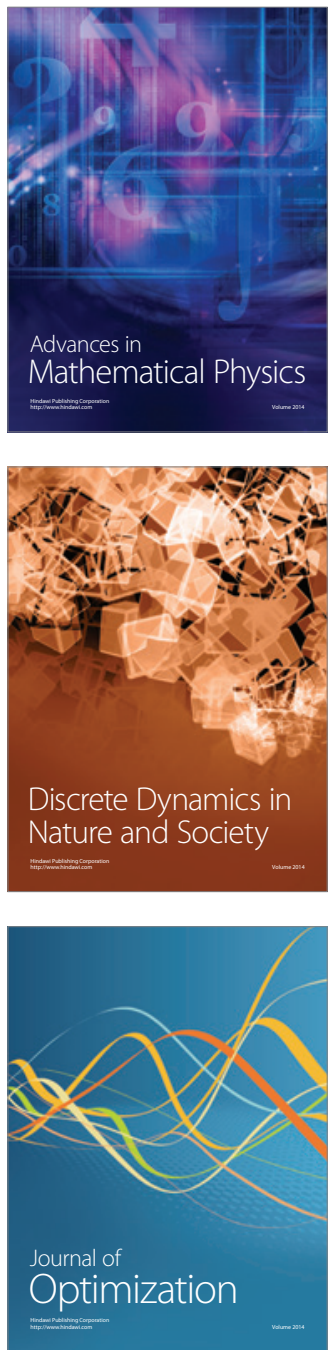